\title{
ALTERNATIVE NETWORK ARCHITECTURES FOR SUPPORTING COMMUNICATIONS FROM THE INTERNATIONAL SPACE STATION
}

\author{
Alex T. Nguyen, Michael Hadjitheodosiou, John. S. Baras \\ Center for Satellite \& Hybrid Communication Networks, \\ Institute for Systems Research, University of Maryland, \\ College Park, MD 20742, USA \\ e-mail: \{alexatn, michalis, baras\}@isr.umd.edu
}

\begin{abstract}
$\underline{\text { ABSTRACT }}$
In order to support the communications needs of the International Space Station (ISS), alternative communications architectures to provide broadband support need to be considered. We address three communications options and evaluate an architecture for the direct to ground option, which could serve as an intermediary solution to satisfy near term communications needs of commercial experiments and payloads on the ISS and overcome certain limitations of the current ISS communications infrastructure. We focus on a particular user's requirements, and examine the system's communications links, and coverage availability. These parameters, along with high-level cost estimates, are compared to using commercial relay satellites, and an enhanced TDRSS. The direct to ground option is viable for store-andforward applications and cost comparable to commercial constellations, but TDRSS is the choice for realtime or continuous data applications.
\end{abstract}

\section{INTRODUCTION}

The continuing deployment of the International Space Station (ISS) has provided a unique opportunity for the scientific and business community to conduct experiments and collect scientific information in space on a serviceable platform.

The platform will include a broad range of research and applications, including microgravity experiments, human physiological studies, space observation, earth observation, and much more. These functions have requirements that can be grouped into sets of similar applications requirements, including:

- Real-time video conferencing,

- Real-time monitoring and control of experiments,

- Downloading of large amounts of data (such as images), and

- On-demand access to data and experiments.

These applications requirements translate into a vast and diverse set of multimedia and data communication requirements, ranging from low to high data rates, ondemand to pre-planned, and low to high coverage. Figure 1 shows a sample matrix of communications requirements and applications that fall into them.

\begin{tabular}{l|ll}
\multicolumn{1}{c}{} & Low coverage & High coverage \\
\cline { 2 - 3 } Low & Monitoring \& & Low rate video, on \\
speed & control & demand data \\
High & Large image & Video \\
speed & download & conferencing \\
\cline { 2 - 3 } & &
\end{tabular}

Figure 1. Sample matrix of communications requirements and applications

\subsection{Motivation / Significance}

In order to achieve these communications services, there will be a need to provide high quality, broadband communications connectivity in order to enable cost effective global access to experimental data from the ISS and other space missions. At the same time, advances in communications technology could allow investigators on Earth to enjoy a virtual presence on board the ISS. However, there are limitations on the current ISS communication system and NASA's TDRSS (Tracking Data Relay Satellite System) that will not satisfy these broad communications needs in the long future.

The current Ethernet onboard the ISS that provides the network backbone for services on the ISS was designed long ago, and does not have the speed necessary to support the new high-demand services. In addition, TDRSS was designed in the 1970's with initially the 
purpose of relaying Tracking, Telemetry, and Control (TT\&C) from NASA satellites to the ground. Its services have worked well, but is becoming increasingly saturated with increased numbers of missions using its services and increased bandwidth requirements.

For these reasons, NASA is investigating alternative long-term solutions for supporting communications from ISS payloads, including the use of commercial technology and commercial assets and infrastructure in space and on the ground. Gradual commercialization of space communications operations could enable:[1]

- Reduction in cost for NASA's and ESA's broadband communication needs;

- Better, faster and easier dissemination of space mission and experimental data if some of the available bandwidth and global coverage of future commercial constellations can be utilized;

- Deployment of next generation commercial satellite constellations (since space agencies might become major customers);

- Faster development in the satellite industry and also enable other commercial entities to take part in experiments and development programs in space, such as future space habitats and planetary missions.

There are a number of research and technology issues that need to be addressed before these services become possible. Among the most important are issues related to:

- $\quad$ Supporting MobileIP

- $\quad$ Supporting security (IPsec)

- Tracking, coverage and antenna technology

- Handover

- Traffic profiles

- Multiple access techniques and network management that allow on-demand access to space data.

\subsection{Approach}

In support of NASA's initiative in evaluating alternative solutions for ISS communications, we have started an effort to investigate the use of next generation commercial satellite constellations for supporting broadband communications for the ISS. several commercial satellite constellations, the existing NASA network and the ground network of candidate commercial constellations. This research work addresses the following topics:

- Identification of potential commercial systems as candidate for investigation, starting from simple GEO (existing) $\mathrm{Ku} / \mathrm{Ka}$-band systems and moving to the next generation $\mathrm{Ka}$ or $\mathrm{V}$ band MEO / LEO systems.

- Development of a detailed simulation model that includes network architecture \& topology of Hybrid Network, and in particular:

- $\quad$ ISS (treated as an extremely LEO satellite) \& ground network.

- Commercial systems' constellation orbit model, ground network topology, information on routing options through constellation, Inter Satellite Links (ISLs) if any.

- Detailed simulation studies to quantify the performance of candidate satellite systems for specific services, protocols \& traffic scenarios and recommend potential design modifications to ensure tele-science QoS requirements are met.

In this paper we list three possible ways we can improve on the current state of the art and thus provide a transition to a system that can deliver higher communication rates to more users and enable a transparent access to space.

We then proceed to analyze one such option, the directto-ground delivery of data from the ISS, in greater detail, as this solution could provide a way to enhance the communications capability in the short term and augment the existing NASA-supported infrastructure. We comment on the economics and cost of doing this as well, since on top of any performance advantages this transition can only happen if it also makes sense from the business point of view.

\section{COMMUNICATION OPTIONS}

Currently, there are three main methods for transmitting data from the ISS to the ground, as shown in Figure 2. These options will be discussed in further detail.

As a first step, we have developed a simulation model for this scenario, consisting of: the ISS, models of 


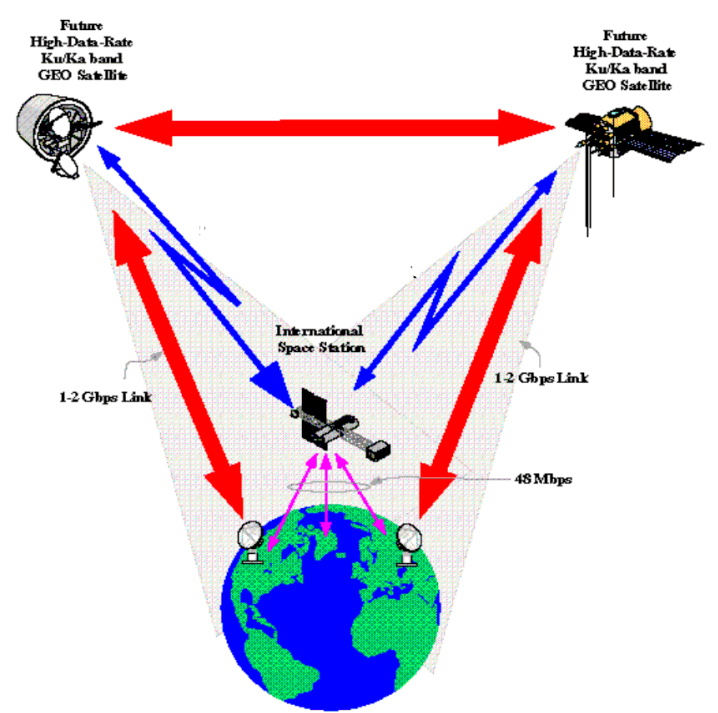

Figure 2. Communications alternatives to/from the ISS

\subsection{Option 1: Using existing TDRSS}

This option is the current communication infrastructure for the ISS, whereby an antenna on the ISS points upward to communicate with one of the TDRSS satellites, which relays the data to the NASA ground terminals.

The Tracking Data Relay Satellite System (TDRSS) consists of 7 satellites in geostationary orbit around the globe that relay data from satellites in Low Earth Orbit (LEO) and Medium Earth Orbit (MEO) to ground facilities at the White Sands Complex in New Mexico, and Guam. The TDR satellites have the capability to forward and return data in the $\mathrm{S}$ and $\mathrm{Ku}$ bands at speeds of up to $300 \mathrm{Mbps}$ in the Ku band.

These systems were developed in the 1970's and have been heavily used over the past two decades. A new generation of TDRS satellites (called TDRS-H, TDRSI, and TDRS-J) has been started to augment the older system and provide additional capacity for users. This new generation TDRS satellite has the additional capability to relay data in Ka-band at up to $300 \mathrm{Mbps}$ without modifications to the ground stations, and up to $800 \mathrm{Mbps}$ with ground station modifications. A new tunable, wideband, high frequency service offered by the 15 -foot antennas provides for the capability of these high data rates. This Ka-Band frequency also establishes interoperability with the international community such as the Europeans and Japanese.[2]

Together, the TDRS satellites provide $100 \%$ coverage for all satellites in LEO orbit, and a very reasonable transmit rate. TDRSS is currently the only system designed to relay communications for fast-moving LEO spacecraft. This makes TDRSS an excellent option to provide communications for the ISS in the long-term future.

Although this system has excellent coverage, its system capacity is being used to its maximum. In addition, there are currently limitations on the main ISS Access Communication System that provides the link to the TDRSS:

- The current design of the ISS high-rate KuBand antenna uses NASA proprietary components, making any future communication system expensive and difficult to implement in a short time.

- Limitations in the current NASA ground network connectivity means that high rate global data dissemination could face significant limitations.

- Many commercial users will need commercially supported broadband communications.

For all these reasons it makes sense to adopt a new uniform architecture that is based on commercial standards to support future commercial services.

\subsection{Option 2: ISS to a commercial satellite constellation acting as relay (In GEO or non- GEO orbit)}

This option essentially means using a commercial fleet of satellites as in lieu of using the TDRSS. There are many Ka-band satellite communication systems that are planned to be deployed within the next few years that provide services such as voice, data, video broadcasting, and many others. This is likely a longterm solution, as there are currently no commercial systems operating at these frequencies that can communicate with moving assets in space. However, if potential interest develops, satellite companies could add a payload to future system expansions that could do that and then offer the relay-to-ground option as a service to NASA or other paying customers.[3]

The intended customers of these services, however, are generally businesses and in some cases, home consumers, not a NASA spacecraft moving in a LEO orbit. Thus, usage of these systems as a relay may not be optimum for the needs of the ISS. These systems will likely be using multiple spot beam antennas pointed towards populated areas of the earth, received 
by either fixed antennas or slow-moving users. Its ability to maintain communications with the ISS traveling at over $17,000 \mathrm{Mph}(27,000 \mathrm{Kph})$ at about 230 miles $(400 \mathrm{~km})$ flying through its hundreds of spot beams may be limited. Coverage is not likely to be nearly as good as that provided by TDRSS, and commercial prices charged by these service providers may be expensive.

\subsection{Option 3: ISS direct to Ka-band ground terminals}

Instead of relaying data through commercial assets in space, the ISS could send the data directly to the ground terminals of satellite companies planning to deploy Kaband satellite systems. The ground networks could be used as access points for downloading ISS data from the ISS Direct-to-Ground (DTG). However, these commercial satellites, as discussed earlier, are generally placed in geostationary orbit for simplicity and to allow customers to downlink from the satellites without having to track the satellites. This means, though, that the ground stations will be comprised of ground terminals that are not capable of tracking, and instead fixed to point towards specific stationary satellites.

Fixed terminals will not be able to track a fast-moving satellite such as the ISS. Due to the possible limited tracking capability, the coverage these terminals provide to a rapidly moving LEO spacecraft might not be sufficient, and there might be a need to either add tracking capability to these terminals or augment the coverage by adding additional terminals distributed globally. The latter is not a likely option considering it would not be an effective cost trade-off.

Because it is uncertain when these commercial systems will actually be realized, new fixed Ka-band terminals could be added to existing NASA ground facilities that are already distributed throughout the globe. These terminals would have tracking capability, and only incur the incremental cost of additional staff and equipment since they would be located at existing facilities. In addition, communications infrastructure on these ground facilities are already in place at these NASA facilities.

In review of these options, we find that the current option of using TDRSS has limitations that do not satisfy requirements of certain potential customers, such as those requiring daily transmissions of images in the order of 1 Terabit. The option of relaying data over commercial in-space assets is a long-term one. While the option of communicating directly to the ground may be a good interim solution, the best may be to consider using NASA's existing ground stations, which is where we will focus on in this paper.

\section{EFFECTS ON COVERAGE FOR ISS DIRECT-TO-GROUND}

We will examine the type of coverage available for using existing NASA facilities augmented with Kaband terminals for ISS communications in the near future, and discuss this option versus using TDRSS and a commercial relay constellation. This will include how the location of the stations (latitude, longitude) affect the coverage, as well as their minimum elevation angle and the ISS antenna's scan angle. We will assume a phased-array antenna onboard the ISS and model it with varying scan angles.

A simulation of the ISS communicating to ground stations was developed using Satellite Tool Kit, which included the ISS with the following properties:

- $400 \mathrm{~km}$

- zero eccentricity circular orbit

- $51.5^{\circ}$ inclination

Included also were the downlink antenna on the ISS and selected NASA ground stations around the world. The scenarios were created with a run time of 10 days in order to minimize aberrations in results. Coverage is defined as the total amount of time the ground stations have contact with the ISS over 24 hours, and was calculated by averaging over the total amount of coverage over the 10-day scenario runs.

\subsection{Latitude \& Longitude}

The latitude and longitude of the stations are first varied, with the stations having zero-degree minimum elevation angles and the ISS onboard antenna having full field of view. Figure 3 shows the distribution of the coverage, which peaks at locations near the inclination angle of the satellite. In this case, the station that has the most coverage is the one placed at \pm 40 degrees latitude. The coverage drops off dramatically for stations placed above the inclination angle, and becomes zero for stations placed at \pm 75 latitude and higher. 
Coverage for Stations Placed at Varying Longitudes

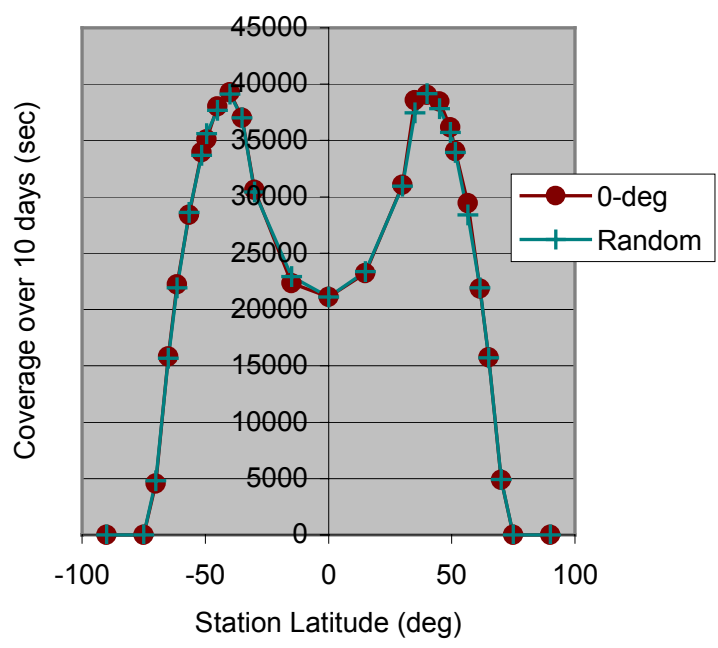

Figure 3. Coverage for varying station lat, long

In addition, the coverage achieved by a station is dependent only on its latitude position and not its longitude. As shown in Figure 3, there is high correlation between stations placed at zero longitude versus random longitude.

\subsection{Minimum Elevation Angle}

To examine the effects of elevation angle, for this scenario, the minimum elevation angle was varied, with the ISS main antenna having scan angle of $90^{\circ}$.
Coverage for Varying Station Elevation Angles

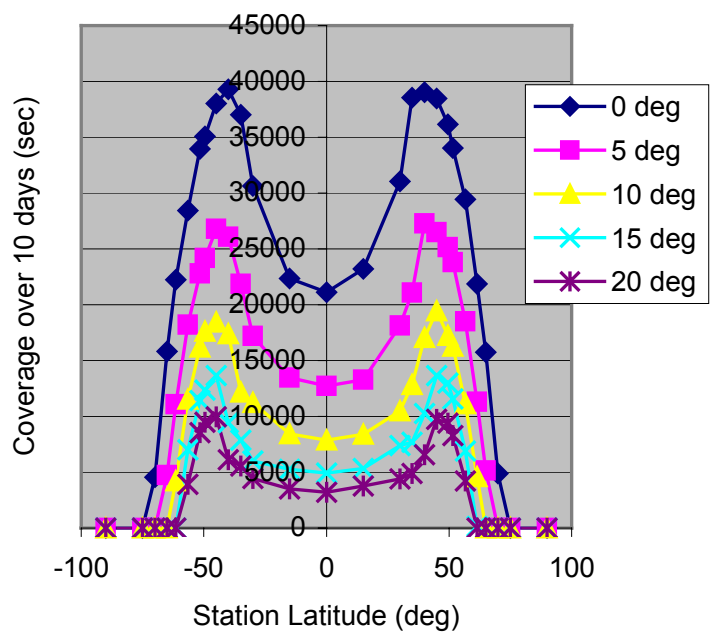

Figure 4. Coverage for varying elevation angle

The results in Figure 4 show that the coverage is almost uniformly reduced at each station as the elevation angle is increased. Also, the location of the peak coverage increases slightly as the minimum elevation is increased because the field of view of the station is reduced, the station must be placed closer to the satellite's inclination in order to provide a more local north contact under the satellite.

For a particular station, the coverage is approximately a logarithmic relationship with respect to minimum elevation angle as shown in Figure 5. In addition, the coverage is more sensitive at lower than at higher minimum elevation angles.

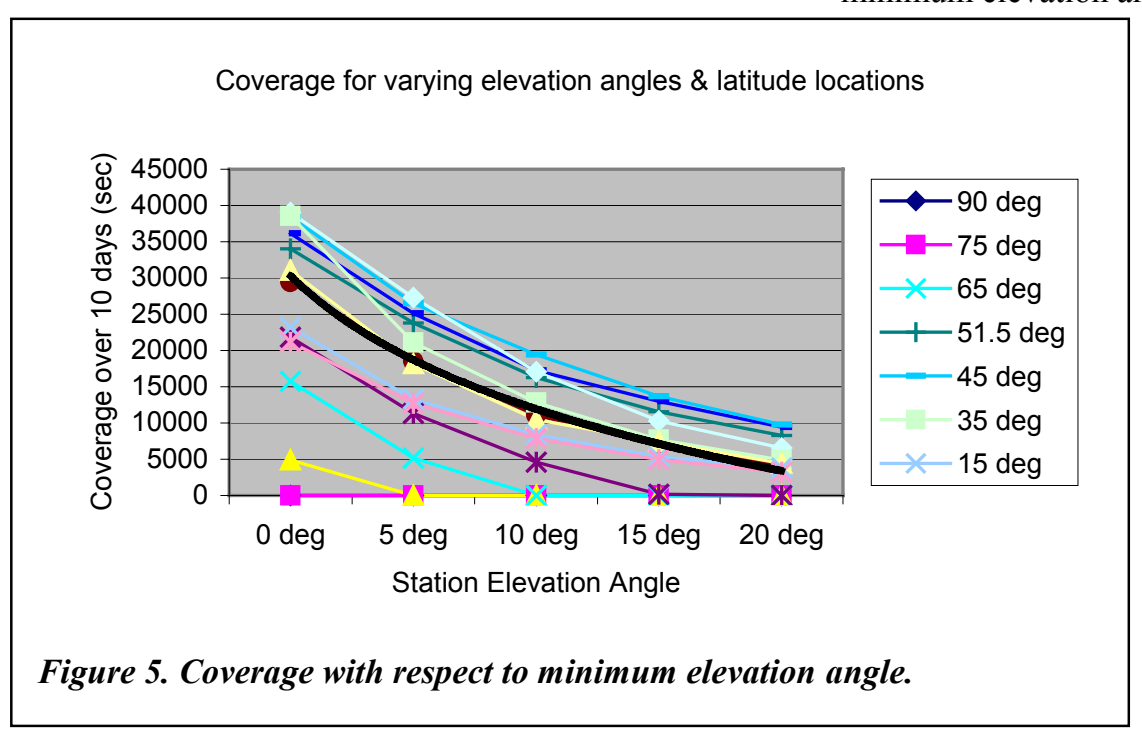

\subsection{Onboard ISS Antenna Scan Angle}

The antenna onboard the ISS is likely to be a phased array antenna, with a certain ability to scan from one edge to the other. In the scenario, the antenna is modeled as a simple cone pointing nadir (towards the center of the earth) with a particular cone half-angle, or scan angle.

To understand the effect of the ISS onboard antenna's scan 
angle on coverage, we fix the locations of the ground stations, and vary the scan angle on the ISS antenna.

Coverage for Varying ISS Antenna Scan Angles

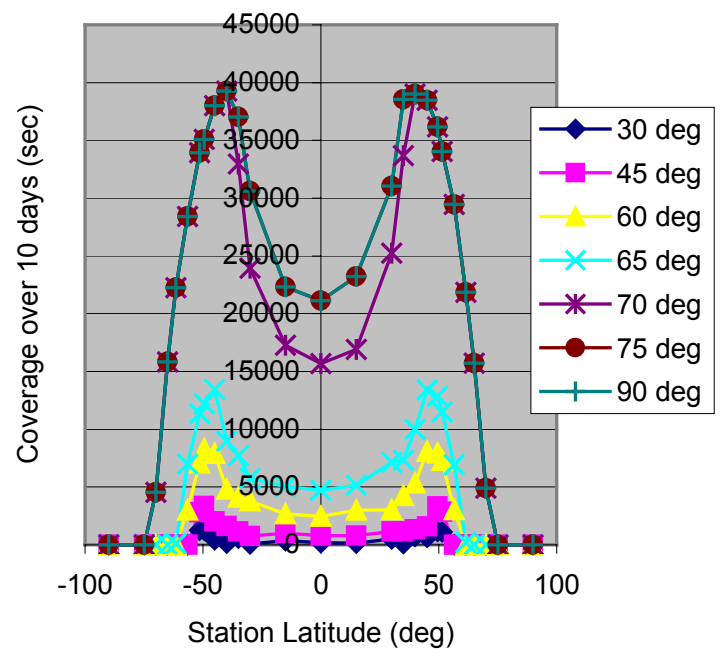

Figure 6. Coverage for varying antenna scan angles.

The best possible coverage can be achieved with an ISS antenna scan angle of about 75 degrees as shown in Figure 6. This is essentially the maximum field of view of the earth at this particular altitude given the curvature of the earth. In addition, the coverage drops off significantly when the cone angle is decreased slightly after 70 degrees, and quickly approaches zero as the cone angle approaches zero. Also, as seen in Figure, there is greater sensitivity when the ISS cone angle is large as compared to when it is small. This indicates that there is significant gain in coverage per degree change in cone angle when the cone angle is

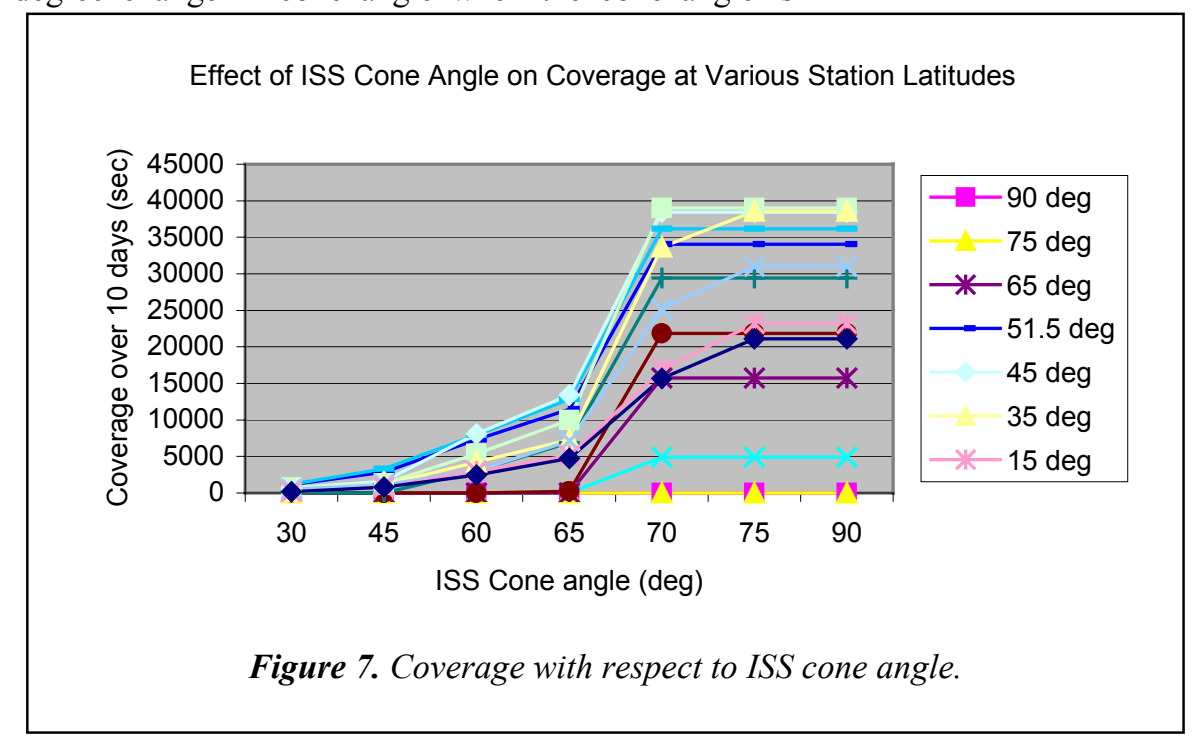

6

American Institute and Aeronautics and Astronautics near 70 degrees. In addition, the coverage reaches its maximum and becomes flat sharply near 70 degrees, but maintains a smooth function all the way up to 90 degrees.

Closer inspection reveals that between 67 degrees and zero degrees cone angle, the curves do not approach the origin, but rather reach zero at a particular point as the cone angle decreases, and stays flat.

\subsection{Elevation Angle and Antenna Scan Angle}

We now examine the variation of coverage with respect to both the ground stations' minimum elevation angle and the ISS antenna scan angle. One station was chosen for this analysis, while its minimum elevation angle and the ISS antenna cone angle was varied.

\section{Coverage for Ground Station Elevation Angle and ISS Antenna Angle}

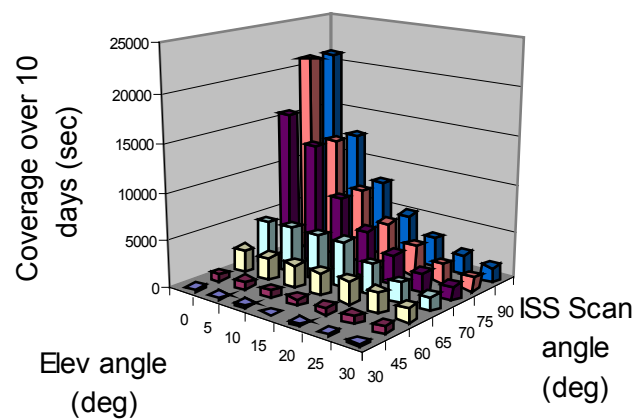

Figure 8. Coverage for varying elevation angle and cone angle.

As seen from Figure 8, there are areas where the coverage is constant for certain elevation angles and ISS cone angles. In general, for a particular minimum station elevation angle, $e$, the coverage unaffected by the ISS cone angle, $\alpha$ until the cone angle is considerably smaller than $90^{\circ}-e$. This is due to the geometry of the ISS antenna and the station as the ISS approaches the station and makes a contact within each other's field of view. In addition, for a particular cone angle, the coverage is 
unaffected by the elevation angle, until the elevation angle is nearly greater than $90^{\circ}-\alpha$. So, there is a boundary between changing and non-changing coverage for various minimum elevation angles and cone angles. Thus, knowing this, we can freely adjust the ISS cone angle and ground station minimum elevation angles for configurations where one does not affect the other.

\section{COVERAGE FOR IMAGING APPLICATION}

The initial design of an ISS direct to ground communication system involves various issues of coverage. These include:

- Antenna power,

- Total coverage availability,

- Duration of each link, and

- Speed of each link.

To determine a first-order coverage capability of the direct-to-ground architecture, we focus on an imaging application on the ISS. An onboard remote sensing device will take images of the surface of the earth or collect other data on or under the earth surface into an image format. The images are temporarily stored onboard the ISS, and downloaded to the ground at the next available ISS contact with a ground station. The application has a minimum requirement of being able to download at least 120 images per day, with each image size about 12 Gbits. These images, which require a total throughput of 1,440 Terabits in every 24-hour period, must be available for commercial customers in the US.

We first determine the ground stations needed to provide this throughput requirement knowing that stations placed near the satellite's inclination provide the best coverage. Stations were chosen closest to the ISS's inclination with minimal overlapping of their coverage cones. Figure 9 shows the placement of the 6 US ground stations providing the best coverage with $10^{\circ}$ minimum elevation angle and ISS antenna scan angle of $60^{\circ}$.

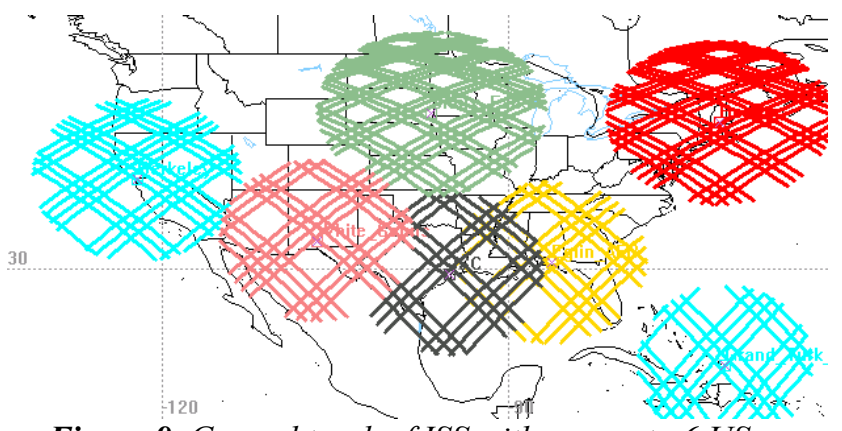

Figure 9. Ground track of ISS with access to 6 US stations.

The stations are ranked in order of best to worst coverage in Table 1. The table also shows the cumulative coverage achieved first for the best station, Sioux Falls, and for adding each subsequent station to the group of stations that can communicate with the ISS.

\begin{tabular}{|c|c|c|c|}
\hline & \multirow{2}{*}{$\begin{array}{l}\text { Individual } \\
\text { Access } \\
\text { (seconds) }\end{array}$} & \multicolumn{2}{|c|}{$\begin{array}{c}\text { Cumulative Access } \\
\text { (seconds) }\end{array}$} \\
\hline & & 10 days & 1 day \\
\hline Sioux_Falls & 6416 & 6,416 & 642 \\
\hline Boston & 5968 & 12,310 & 1,231 \\
\hline Berkeley & 4302 & 16,476 & 1,648 \\
\hline White_Sands & 3723 & 19,973 & 1,997 \\
\hline Eglin_AFB & 3633 & 23,984 & 2,398 \\
\hline JSC & 3585 & 25,630 & 2,563 \\
\hline
\end{tabular}

Table 1. Coverage of stations.

From this data, we can determine the best stations to use by calculating the throughput for using the best station, and adding each subsequent station until the desired throughput is achieved. Table 2 shows the amount of throughput achieved with each incremental station in Gigabits per day for transmit speeds of 180 Mbps, $361 \mathrm{Mbps}$, and $622 \mathrm{Mbps}$. If each downloaded image size was $12 \mathrm{~Gb}$, the table also shows the number of files that can be downloaded in a 24-hour period.

\begin{tabular}{|l|r|r|r|r|r|r|}
\hline & \multicolumn{3}{|c|}{$\begin{array}{c}\text { Data Throughput (Gb) } \\
\text { for specified transmit } \\
\text { rates (Gbps) }\end{array}$} & $\begin{array}{r}\text { \# Image downloads for } \\
\text { Image size 12 Gb }\end{array}$ \\
\cline { 2 - 7 } & $\begin{array}{r}180 \\
\text { Mbps }\end{array}$ & $\begin{array}{r}361 \\
\text { Mbps }\end{array}$ & $\begin{array}{r}622 \\
\text { Mbps }\end{array}$ & $\begin{array}{r}180 \\
\text { Mbps }\end{array}$ & $\begin{array}{r}361 \\
\text { Mbps }\end{array}$ & $\begin{array}{r}622 \\
\text { Mbps }\end{array}$ \\
\hline Sioux_Falls & 115 & 232 & 399 & 9 & 19 & 33 \\
\hline Boston & 222 & 444 & 766 & 18 & 37 & 63 \\
\hline Berkeley & 297 & 595 & 1,025 & 24 & 49 & 85 \\
\hline White_Sands & 360 & 721 & 1,242 & 29 & 60 & 103 \\
\hline Eglin_AFB & 432 & 866 & 1,492 & 35 & 72 & 124 \\
\hline JSC & 461 & 925 & 1,594 & 38 & 77 & 132 \\
\hline
\end{tabular}

Table 2. Throughput for stations.

7 
Thus, using a direct to ground architecture does provide enough coverage for a typical store-and-forward application on the ISS.

\section{COST \& COMPARISONS WITH OTHER ARCHITECTURES}

\subsection{Cost of Direct-to-Ground}

In order to determine a cost comparison between the direct-to-ground option and other communications architectures, we look in this section at the cost components that vary among these communication alternatives. Not considered in these cost estimates are the data processing center for the instrument, the payload operations center, the instrument itself, or other operating costs since these are assumed to be the same for all architectures.

Because there are already over 200 ground stations at over 50 NASA facilities distributed at strategic locations throughput the globe, we will only consider installing additional Ka-band terminals on existing NASA ground facilities. This saves the cost of having to build new facilities, and only incurs the incremental cost of adding additional terminals and staff to operate and maintain the terminals.

The cost for space hardware is calculated using cost estimating relationships (CERs) that are based on empirical data and is generally a function of mass.[4] We assume a mass for the antenna of about $50 \mathrm{~kg}$, and a mass for the communications electronics of about 40 $\mathrm{kg}$. An additional 1.2 factor is applied to the research, development, testing, and evaluation (RDT\&E) cost to account for additional technological complexity for Kaband phased array antenna equipment compared to other common equipment. Because this is a phased array antenna, it may have less weight than a conventional antenna which has gimballing mechanisms to enable tracking. The cost for RDT\&E of the ISS direct to ground antenna and corresponding electronics is approximately $\$ 26.8$ million, while production of the communication system is about $\$ 9.5$ million.

The ISS downlink antenna and corresponding electronics will be launched by the Space Shuttle, which has a cost of bringing payloads into orbit of nearly $\$ 10,000$ per $\mathrm{kg}$. In addition, a cost is added to account for insurance during the launch, and for the payload itself that are based on a percentage of the launch and payload cost respectively. The total cost of the Launch segment is about \$2 million.
The cost for the ground segment of a satellite communication system can be generally broken down into elements with the percentage breakdowns based on data from other satellite ground systems and modified slightly.[4] The cost for the equipment of each ground station includes the antenna and transmit / receive equipment for the station, which is all estimated to be about $\$ 200,000$. The remaining ground segment costs are calculated as relative percentages of the equipment cost.

Because there are only a few ground terminals and the cost of these terminals do not greatly impact the overall cost, we will ignore a learning curve. The cost for establishing each ground station is approximately $\$ 606,000$.

Running each ground station will include staff to operate and manage the station, regular maintenance, and communications lines to the Internet backbone. Because these terminals will be built on existing NASA facilities, only the incremental cost of additional staff and maintenance is incurred. The maintenance cost will be taken as a percentage $(10 \%)$ of the ground station equipment, software, and facilities. In addition, communications links such as a T1 line for data and PSTN for voice will be needed, and is based on surveys of prices for such services. Communications costs for facilities located outside the US may incur additional inter-continental data lines. It costs approximately $\$ 230,000$ to operate and maintain each ground station each year.

\begin{tabular}{|c|c|c|}
\hline Space Segment Costs & & \\
\hline ISS onboard antenna RDT\&E & $\$$ & 26,802 \\
\hline ISS onboard antenna Prod & $\$$ & 9,493 \\
\hline Program Level & $\$$ & 9,649 \\
\hline Ground Segment Costs & & \\
\hline Ground Station Elements & $\$$ & 2,400 \\
\hline System Level & $\$$ & 1,236 \\
\hline Launch Segment Costs & & \\
\hline Shuttle Launch & $\$$ & 819 \\
\hline Insurance & $\$$ & 1,031 \\
\hline Total Initial Development & $\$$ & 51,429 \\
\hline
\end{tabular}

Assuming amortization in 3 years and the daily throughput calculated earlier, the direct-to-ground system is estimated to cost the owner about $\$ .25$ per Megabyte during the first 3 years. This is the cost to the owner of the system for researching and building his own system to downlink data from the ISS imaging 
instrument. If there is excess capacity, it could be leased to potential customers at a price higher than this, possibly at approximately $\$ .40$ per Megabyte.

\subsection{Comparison}

Overall, as shown in Table 4, the Direct to Ground architecture is suitable for store-and-forward applications that do not require large amounts of coverage. The TDRSS is the best option to use for video conferencing type applications due to its continuous coverage. While the Direct to Ground option offers flexibility, using a commercial relay system may allow easier setup and less initial cost investment.

\begin{tabular}{|c|c|c|c|}
\hline & Direct to Ground & $\begin{array}{l}\text { Commercial } \\
\text { Relay }\end{array}$ & TDRSS (Ku) \\
\hline 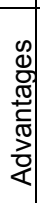 & $\begin{array}{l}\text { Flexible, } \\
\text { Scalable, } \\
\text { Prices competitive } \\
\text { Good for store- } \\
\text { and-forward } \\
\text { applications }\end{array}$ & $\begin{array}{l}\text { Very little } \\
\text { system setup } \\
\text { required }\end{array}$ & $\begin{array}{l}100 \% \text { coverage, } \\
\text { Good for real- } \\
\text { time or on } \\
\text { demand } \\
\text { applications }\end{array}$ \\
\hline 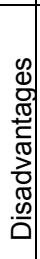 & $\begin{array}{l}\text { Not good for real- } \\
\text { time applications, } \\
\text { Requires additional } \\
\text { building of facilities }\end{array}$ & $\begin{array}{l}\text { Prices and } \\
\text { entire system } \\
\text { uncertain, } \\
\text { Systems not } \\
\text { tailored to } \\
\text { needs of ISS } \\
\text { customers }\end{array}$ & $\begin{array}{l}\text { Possible slow } \\
\text { ISS onboard } \\
\text { communications } \\
\text { system, } \\
\text { Capacity for } \\
\text { commercial } \\
\text { applications may } \\
\text { be limited later }\end{array}$ \\
\hline
\end{tabular}

Table 4. Comparison of three architectures. [5]

\begin{tabular}{|l|l|l|l|}
\hline & $\begin{array}{l}\text { Direct to } \\
\text { Ground }\end{array}$ & Astrolink & $\begin{array}{l}\text { TDRSS } \\
(\mathbf{K u})\end{array}$ \\
\hline $\begin{array}{l}\text { Cost per } \\
\text { Megabyte }\end{array}$ & $\$ 0.25$ & $\$ 0.05-\$ 0.5$ & $\begin{array}{l}\$ 0.09- \\
\$ 0.13\end{array}$ \\
\hline $\begin{array}{l}\text { Transmit } \\
\text { rate }\end{array}$ & $622 \mathrm{Mbps}$ & $\begin{array}{l}\text { Up to } 110 \\
\text { Mbps }\end{array}$ & $300 \mathrm{Mbps}$ \\
\hline
\end{tabular}

Table 5. Cost per Megabyte for three architectures. [5],[6]

In comparison with the commercial relay system, the direct to ground option offers about the same amount of coverage at similar costs. As shown in Table 5, the TDRSS offers complete coverage, at relatively low cost for the $\mathrm{Ku}$ band satellites. The new Ka band satellites will likely be slightly more costly.[6]

\section{SUMMARY \& FUTURE WORK}

We are in the process of expanding the model to develop the framework for handover / connectivity support analysis and plan to continue developing the simulation platform to:
- Perform end-to-end optimization \& suggest solutions to support particular protocols or QoS requirements for specific services over the space-to-ground link,

- Investigate traffic characteristics of particular services and find ways to optimize dynamic resource / capacity sharing that would maximize revenue,

- Analyze the business case study and explore ways to maximize revenue by 1) estimating the bandwidth cost of this commercial service, and 2) investigating dynamic pricing solutions for different customers.

Additional studies can be performed for other classes of systems besides the ISS. This could include user spacecraft with various types of antennas, and various types of orbits (such as LEO, MEO) and various classes of commercial relay constellations.

In addition, the coverage results could be tied to RF and other communication protocol studies. Effects on changing antenna size and power, for example, would have an impact on the antenna cone angle used in these studies. Optimum antenna choices could be derived from a combination of these studies.

Finally, with additional information such as pricing and cost estimates of commercial systems, a more sophisticated system could be developed with dynamic pricing. While NASA uses a rather flat pricing structure for its use of TDRSS (generally priced in dollars per minute), commercial systems could be based on various market conditions. Prices could be dynamically driving by the time (of day, month, or year), level of demand, amount of competition, level of service requested and frequency of service, and many other factors. Monte Carlo simulations could be conducted to view the effects of these changing market conditions and observe how pricing may be competitive with TDRSS and other communications architectures.

\section{REFERENCES}

[1] Hadjitheodosiou, M., Nguyen, A, "Extending IP Services to Space Missions", Proc. 19th AIAA ICSSc, Toulouse, France, April 2001.

[2] NASA, "TDRS Antennas" [online] (http://nmsp.gsfc.nasa.gov/tdrss/ant.html)

[3] Hadjitheodosiou, M., Nguyen, A., "Commercial Communication Satellite Constellations Carrying Traffic From NASA 
Missions", Proc. 18th AIAA ICSSC, April 2000.

[4] Larson, Wiley J. and Wertz, James R. Space Mission Analysis and Design, second edition. Microcosm, Inc. (Torrance: 1992).

[5] Nguyen, Alex, "A Direct to Ground Architecture for supporting Communications for the ISS", M.S. Thesis, University of Maryland, Dec. 2001.

[6] E. Lutz, M. Werner, A. Jahn, Satellite Systems for Personal and Broadband Communications. Springer, 2000
[7] Hadjitheodosiou, M., Nguyen, A. "Novel Ways to Provide Communication Support for Future Space Missions", Proc. GLOBECOM2000, Nov. 2000.

[8] Nguyen, A.T., Zhou, x., Hadjitheodosiou, M., Baras, J.S., "A direct-to-ground architecture for supporting Commercial Communications from the International Space Station" Submitted, IEEE ICC 2002, New York, NY, Apr. 2002.

Acknowledgements: This work is supported by the Center for Satellite and Hybrid Communication Networks, under NASA cooperative agreement NAG59150. 